\title{
Constraints on cosmological parameters in power-law cosmology
}

\author{
Sarita Rani ${ }^{1}$, A. Altaibayeva ${ }^{2}$, M. Shahalam ${ }^{3}$, J. K. \\ Singh $^{1}$, R. Myrzakulov ${ }^{2}$
}

${ }^{1}$ Netaji Subhas Institute of Technology, University of Delhi, New Delhi, India

${ }^{2}$ Department of General and Theoretical Physics, Eurasian National University, Astana, Kazakhstan

${ }^{3}$ Center For Theoretical Physics, Jamia Millia Islamia, New Delhi, India

E-mail:

sarita_maths@yahoo.co.in, aziza.bibol@mail.ru,mdshahalam@ctp-jamia.res.in, jainendrrakumar@rediffmail.com,rmyrzakulov@gmail.com

\begin{abstract}
In this paper, we examine observational constraints on the power law cosmology; essentially dependent on two parameters $H_{0}$ (Hubble constant) and $q$ (deceleration parameter). We investigate the constraints on these parameters using the latest 28 points of $\mathrm{H}(\mathrm{z})$ data and 580 points of Union2.1 compilation data and, compare the results with the results of $\Lambda \mathrm{CDM}$. We also forecast constraints using a simulated data set for the future JDEM, supernovae survey. Our studies give better insight into power law cosmology than the earlier done analysis by Kumar [arXiv:1109.6924] indicating it tuning well with Union2.1 compilation data but not with $\mathrm{H}(\mathrm{z})$ data. However, the constraints obtained on $\left\langle H_{0}\right\rangle$ and $\langle q\rangle$ i.e. $H_{0}$ average and $q$ average using the simulated data set for the future JDEM, supernovae survey are found to be inconsistent with the values obtained from the $\mathrm{H}(\mathrm{z})$ and Union2.1 compilation data. We also perform the statefinder analysis and find that the power-law cosmological models approach the standard $\Lambda$ CDM model as $q \rightarrow-1$. Finally, we observe that although the power law cosmology explains several prominent features of evolution of the Universe, it fails in details.
\end{abstract}

Keywords: inflation, dark energy theory 


\section{Contents}

1 Introduction 1

2 Power Law Cosmology 2

$\begin{array}{lll}3 & \Lambda \mathrm{CDM} & 3\end{array}$

4 Observational Constraints $\quad 5$

4.1 Constraints on Statefinders 9

5 Conclusion $\quad 10$

\section{Introduction}

The Standard Cosmological Model (SM) of Universe a la $\Lambda$ CDM complemented by the inflationary phase is remarkably a successful theory, although, the cosmological constant problem still remains to be one of the major unsolved problems [1] of our times. It is therefore reasonable to examine the alternative cosmological models to explain the observed Universe. Power-law cosmology is one of the interesting alternatives to deal with some usual problems (age, flatness and horizon problems etc.) associated with the standard model. In such a model, the cosmological evolution is explained by the geometrical scale factor $a(t) \propto t^{\beta}$ with $\beta$ as a positive constant. The power law evolution with $\beta \geq 1$ has been discussed at length in a series of articles in distinct contexts [2-10]; phantom power-law cosmology is discussed in reference [14]. The motivation for such a scenario comes from a number of considerations. For example, power-law cosmology does not face the horizon problem [9], as well as the flatness problem. Another remarkable feature of these models is that they easily accommodate high redshift objects and hence reduce the age problem. These models also deal with the fine tuning problem, in an attempt to dynamically solve the cosmological constant problem [15-19].

A power law evolution of the cosmological scale factor with $\beta \approx 1$ is an excellent fit to a host of cosmological observations. Any model supporting such a coasting presents itself as a falsifiable model as far as classical cosmological tests are concerned as it exhibits distinguishable and verifiable features. Classical cosmological tests also support such kind of evolution, such as the galaxy number counts as a function of redshift and the data on angular diameter distance as a function of redshift [20]. However, these tests are not considered as reliable tests of a viable model since these are marred by evolutionary effects (e.g. mergers). Now, SNe Ia (reliable standard candles), and hubble test have become more reliable to that of a precision measurement.

Cosmological parameters prove to be the backbone of any of the cosmological models, therefore it becomes important to obtain a concise range or more specifically, the estimated values of such parameters using available observational data, so that the 
said model can explain the present evolution of Universe more precisely. In this series of cosmological parameters we observe that Hubble constant $\left(H_{0}\right)$ and deceleration parameter $(q)$ are very important in describing the current nature of the Universe. $H_{0}$ explains the current expansion rate of the Universe whereas $q$ describes the nature of the expansion rate. In last few years, various attempts have been done to evaluate the value of $H_{0}$. Freedman et al. [21] evaluated a value of $H_{0}=72 \pm 8 \mathrm{~km} / \mathrm{s} / \mathrm{Mpc}$. Suyu et al.[22] evaluated $H_{0}$ as $69.7_{-5.0}^{+4.9} \mathrm{~km} / \mathrm{s} / \mathrm{Mpc}$. WMAP7 evaluated the value of $H_{0}=71.0 \pm 2.5$ $\mathrm{km} / \mathrm{s} / \mathrm{Mpc}$ (with WMAP alone), and $H_{0}=70.4_{-1.4}^{+1.3} \mathrm{~km} / \mathrm{s} / \mathrm{Mpc}$ (with Gaussian priors ) [23]. Numerous other evaluates of $H_{0}$ are $73.8 \pm 2.4 \mathrm{~km} / \mathrm{s} / \mathrm{Mpc}$ [24], $67.0 \pm 3.2$ $\mathrm{km} / \mathrm{s} / \mathrm{Mpc}$ [25]. Most recent PLANCK evaluate of the Hubble constant gives a value of $H_{0}=67.3 \pm 1.2 \mathrm{~km} / \mathrm{s} / \mathrm{Mpc}$ [26]. Along with the above mentioned evaluates of $H_{0}$, several other authors, [7], [9-13] obtained the constraints on cosmological parameters including $H_{0}, q$ and $\beta$ for open, closed and flat power law cosmology. Numerical results for flat power-law cosmology have been described in Table 2.

In a recent paper, Kumar [12] has investigated observational constraints on the power-law cosmological parameters using $\mathrm{H}(\mathrm{z})$ and SN Ia data and discussed various features of power-law cosmology. In the present work, we are investigating the scenario similar to an analysis done in reference [12] for flat power law cosmology. We compare the results of the model under consideration with the results obtained from $\Lambda \mathrm{CDM}$ and with that of Kumar [12]. We use the most recent observational datasets such as 28 points of $\mathrm{H}(\mathrm{z})$ data [27] and Union2.1 compilation (SN) data [28] (taking into account the full covariance matrix). Here, we also forecast constraints using a simulated data set for the future JDEM, supernovae survey [36, 37] and also employ Statefinder analysis of the results obtained.

\section{Power Law Cosmology}

For a flat FLRW metric, the line element is

$$
d s^{2}=c^{2} d t^{2}-a^{2}(t)\left[d r^{2}+r^{2}\left(d \theta^{2}+\sin ^{2} \theta d \phi^{2}\right)\right],
$$

where $a(t)$ is the scale factor and $t$ is the cosmic proper time. In this paper, we discuss general power law cosmology,

$$
a(t)=a_{0}\left(\frac{t}{t_{0}}\right)^{\beta} .
$$

Here, $t_{0}$ and $\beta$ represent the present age of Universe and dimensionless positive parameter respectively. Here and subsequently, the subscript 0 defines the present-day value of the parameters considered.

$$
H=\frac{\dot{a}}{a}=\frac{\beta}{t},
$$

and

$$
H_{0}=\frac{\beta}{t_{0}}
$$


The relation between the red shift and the scale factor is given by

$$
\frac{a(t)}{a_{0}}=\frac{1}{1+z}
$$

The age of Universe at redshift $z$ is given as

$$
t(z)=\frac{\beta}{H(z)},
$$

where,

$$
H(z)=H_{0}(1+z)^{\frac{1}{\beta}} .
$$

The acceleration of Universe can be measured through a dimensionless cosmological function called as the deceleration parameter $q$. In this scenario $q$ is

$$
q=-\frac{\ddot{a}}{a H^{2}}=\frac{1}{\beta}-1
$$

where, $q<0$ explains an accelerating Universe, whereas $q \geq 0$ describes a Universe which is either decelerating or expanding at the 'coasting' rate. Equation (2.5) in terms of $q$ can be written as

$$
H(z)=H_{0}(1+z)^{(1+q)} .
$$

Equation (2.7) implies that the parameters $H_{0}$ and $q$ explain history of the Universe in power law cosmology. In this paper, we study the well behaved power-law cosmological model, focusing on the parameters $q$ and $H_{0}$, also we find the observational constraints on both of the above parameters to the latest 28 data points of $\mathrm{H}(\mathrm{z})$ [27] and Union2.1 compilation data [28] of 580 points. We also use the simulated data for upcoming Supernova (SN) surveys like JDEM to constrain the above said parameters $[36,37]$.

\section{$3 \quad \Lambda \mathrm{CDM}$}

As per the point of view of cosmology, the most simplest candidate of dark energy is the cosmological constant whose energy density remains constant with time i.e. $\rho_{\Lambda} \equiv \frac{\Lambda}{8 \pi G}=-p_{\Lambda}$ and its equation of state is, $\omega_{\Lambda}=-1$. A Universe having matter in the form of dust and $\Lambda$ is known as $\Lambda$ CDM. In flat FLRW Universe, Hubble parameter for $\Lambda \mathrm{CDM}$ model has the following form:

$$
H(z)=H_{0}\left[\Omega_{0 m}(1+z)^{3}+\left(1-\Omega_{0 m}\right)\right]^{1 / 2} .
$$

Here, $\Omega_{0 m}$ and $H_{0}$ are the present matter density and Hubble parameters respectively. 


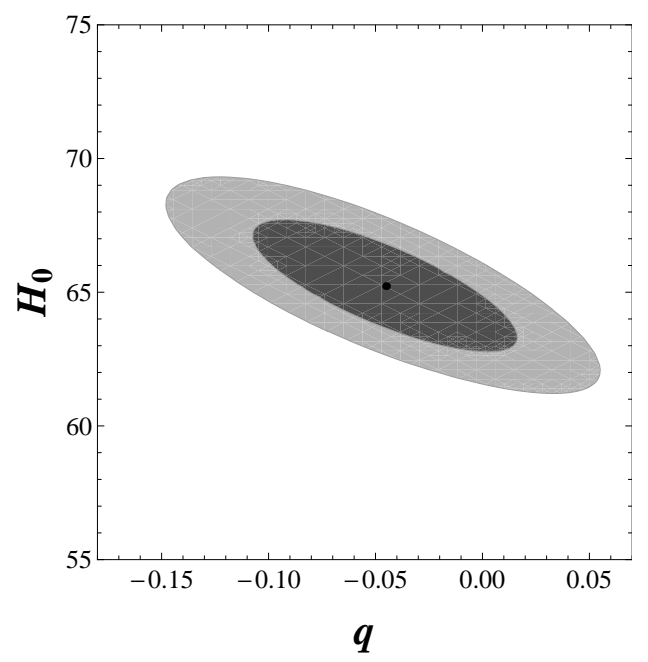

(a)

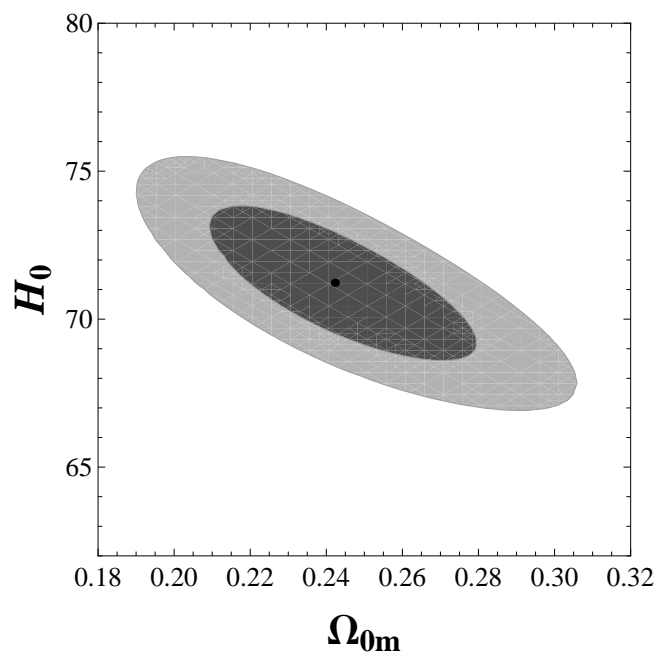

(b)

Figure 1. This figure corresponds to the latest $\mathrm{H}(\mathrm{z})$ data. The panels (a) and (b) show the $1 \sigma$ (dark shaded) and $2 \sigma$ (light shaded) likelihood contours in the $q-H_{0}$ and $\Omega_{0 m}-H_{0}$ planes, obtained for power-law and $\Lambda \mathrm{CDM}$ models respectively. The $H_{0}$ is represented in unit of $\mathrm{Km} / \mathrm{s} / \mathrm{Mpc}$. Black dots designate the best fit values of the respective parameters.

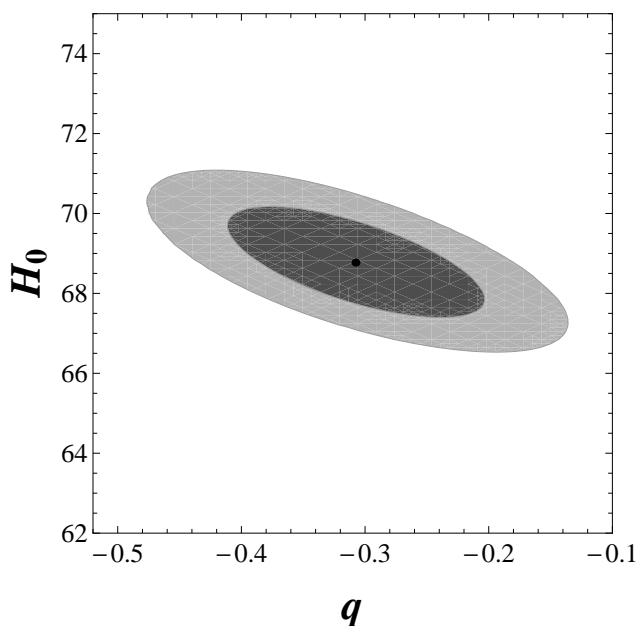

(a)

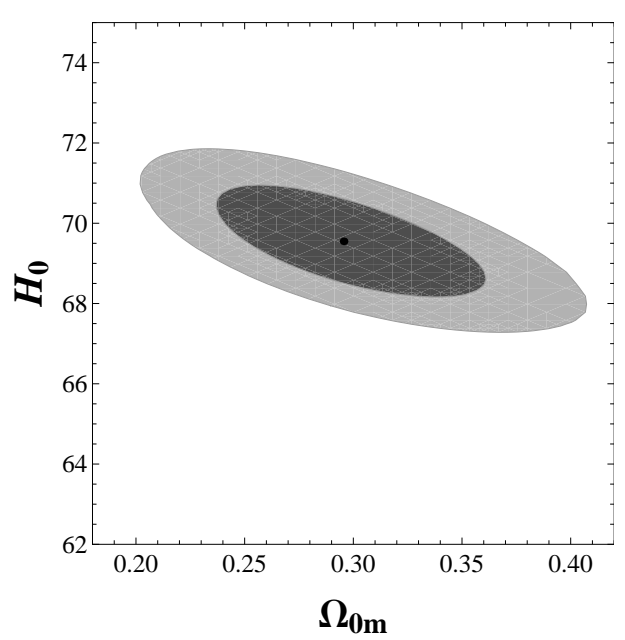

(b)

Figure 2. This figure corresponds to SN data. The panels (a) and (b) show the $1 \sigma$ (dark shaded) and $2 \sigma$ (light shaded) likelihood contours in the $q-H_{0}$ and $\Omega_{0 m}-H_{0}$ planes, obtained for power-law and $\Lambda \mathrm{CDM}$ models respectively. Here, the unit of $H_{0}$ is $\mathrm{Km} / \mathrm{s} / \mathrm{Mpc}$. Black dots represent the best fit values of the respective parameters. 


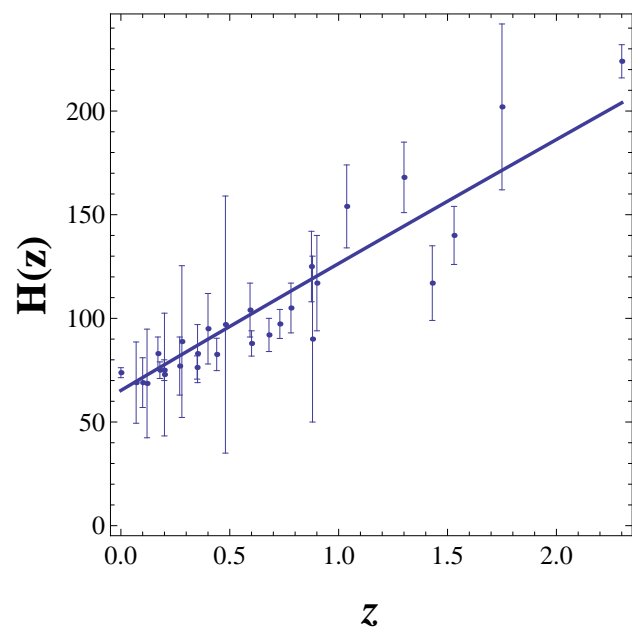

(a)

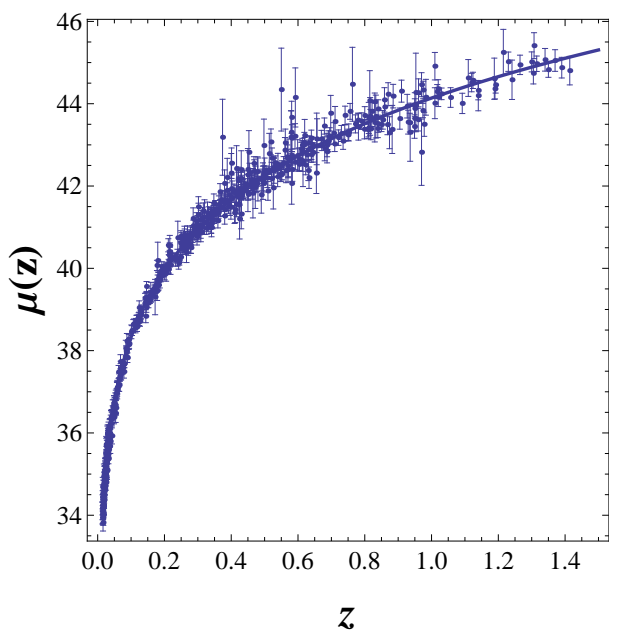

(b)

Figure 3. Panels (a) and (b) correspond to the latest H(z) data and SN data respectively, with error bars. In both the panels, solid line shows the best fitted behaviour for power law cosmological model. The $\mathrm{H}(\mathrm{z})$ is expressed in unit of $\mathrm{Km} / \mathrm{s} / \mathrm{Mpc}$. For panel (b), we have used diagonal covariance matrix.

\section{Observational Constraints}

- $\mathrm{H}(\mathrm{z})$ Data:

We find the observational constraints on both of the parameters $H_{0}$ and $q$ to the latest 28 data points of $\mathrm{H}(\mathrm{z})[27]$ in the redshift range $0.07 \leq z \leq 2.3$. The values are presented in the Table 1 . To complete the data set, we use $H_{0}$, as estimated in the reference [24].

we define $\chi^{2}$ as

$$
\chi_{H}^{2}=\sum_{j=1}^{29} \frac{\left(H_{e x p}\left(z_{j}\right)-H_{o b s}\left(z_{j}\right)\right)^{2}}{\sigma_{j}^{2}} .
$$

Where $H_{\text {exp }}$ is the expected value of the Hubble parameter, $H_{o b s}$ is the observational value and, $\sigma_{j}$ is the corresponding $1 \sigma$ error. The power law cosmological model contains two independent parameters namely $q$ and $H_{0}$. As $\beta>0$ is required in power law cosmology, hence $q>-1$ and $H_{0} \geq 0$, therefore we find the best fit values of $q$ and $H_{0}$ by restricting the parametric space as $q>-1$ and $H_{0} \geq 0$. As a result, we obtain the best fit values of the parameters as $q=-0.0451, H_{0}=65.2299 \mathrm{~km} / \mathrm{s} / \mathrm{Mpc}$ and $\chi_{\delta}^{2}=1.8131$, and the values of the parameters with $1 \sigma$ error are obtained as $q=-0.0451_{-0.0625}^{+0.0614}$ and $H_{0}=65.2299_{-2.4607}^{+2.4862} \mathrm{~km} / \mathrm{s} / \mathrm{Mpc}$, where $\chi_{\delta}^{2}=\chi_{\min }^{2} /($ degree of freedom). We carry out the same analysis for $\Lambda \mathrm{CDM}$ model and as a result, the best fit values of the parameters are obtained as $\Omega_{0 m}=0.2422, H_{0}=71.2272 \mathrm{~km} / \mathrm{s} / \mathrm{Mpc}$ and 
Table 1. $H(z)$ measurements (in unit $\left[\mathrm{km} \mathrm{s}^{-1} \mathrm{Mpc}^{-1}\right]$ ) and their errors [27].

\begin{tabular}{|c|c|c|c|}
\hline$z$ & $\begin{array}{c}H(z) \\
(\mathrm{km} / \mathrm{s} / \mathrm{Mpc})\end{array}$ & $\begin{array}{c}\sigma_{H} \\
(\mathrm{~km} / \mathrm{s} / \mathrm{Mpc})\end{array}$ & Reference \\
\hline 0.070 & 69 & 19.6 & [29] \\
\hline 0.100 & 69 & 12 & {$[30]$} \\
\hline 0.120 & 68.6 & 26.2 & [29] \\
\hline 0.170 & 83 & 8 & {$[30]$} \\
\hline 0.179 & 75 & 4 & [31] \\
\hline 0.199 & 75 & 5 & [31] \\
\hline 0.200 & 72.9 & 29.6 & [29] \\
\hline 0.270 & 77 & 14 & {$[30]$} \\
\hline 0.280 & 88.8 & 36.6 & [29] \\
\hline 0.350 & 76.3 & 5.6 & {$[32]$} \\
\hline 0.352 & 83 & 14 & [31] \\
\hline 0.400 & 95 & 17 & [30] \\
\hline 0.440 & 82.6 & 7.8 & [33] \\
\hline 0.480 & 97 & 62 & [34] \\
\hline 0.593 & 104 & 13 & [31] \\
\hline 0.600 & 87.9 & 6.1 & [33] \\
\hline 0.680 & 92 & 8 & [31] \\
\hline 0.730 & 97.3 & 7.0 & [33] \\
\hline 0.781 & 105 & 12 & [31] \\
\hline 0.875 & 125 & 17 & [31] \\
\hline 0.880 & 90 & 40 & [34] \\
\hline 0.900 & 117 & 23 & {$[30]$} \\
\hline 1.037 & 154 & 20 & [31] \\
\hline 1.300 & 168 & 17 & [30] \\
\hline 1.430 & 177 & 18 & [30] \\
\hline 1.530 & 140 & 14 & {$[30]$} \\
\hline 1.750 & 202 & 40 & [30] \\
\hline 2.300 & 224 & 8 & [35] \\
\hline
\end{tabular}

$\chi_{\delta}^{2}=0.7174$, and the values of the parameters with $1 \sigma$ error are obtained as $\Omega_{0 m}=0.2422_{-0.0331}^{+0.0373}$ and $H_{0}=71.2272_{-2.6291}^{+2.6112} \mathrm{~km} / \mathrm{s} / \mathrm{Mpc}$. By comparing the values of $\chi_{\delta}^{2}$ of both the models, we find that the power law cosmological model does not fit well to the latest $\mathrm{H}(\mathrm{z})$ data. The $1 \sigma$ (dark shaded) and $2 \sigma$ (light shaded) likelihood contours for both the models are shown in figure 1 . Best fitted behaviour for power law cosmological model with $\mathrm{H}(\mathrm{z})$ data and error bars has been shown in panel (a) of figure 3. It is worthwhile to notice from Table 2 that the best fit value of $q=-0.18$ [12] has been significantly shifted to $q=-0.0451$ with latest $\mathrm{H}(\mathrm{z})$ data. It was claimed in reference [12] that power law model fits 
Table 2. Summary of the numerical results for flat power law cosmological model.

\begin{tabular}{lcccr}
\hline \hline Data & $q$ & $H_{0}(\mathrm{~km} / \mathrm{s} / \mathrm{Mpc})$ & $\beta$ & Refs. \\
\hline$H(z)$ (14 points) & $-0.18_{-0.12}^{+0.12}$ & $68.43_{-2.80}^{+2.84}$ & - & \\
SN (Union2) & $-0.38_{-0.05}^{+0.05}$ & $69.18_{-0.54}^{+0.55}$ & - & {$[12]$} \\
\hline WMAP7 & - & $70.3_{-2.5}^{+2.5}$ & $0.99_{-0.04}^{+0.04}$ & {$[13]$} \\
WMAP7+BAO+H(z) & - & $70.4_{-1.4}^{+1.4}$ & $0.99_{-0.02}^{+0.02}$ & \\
\hline H( $z$ ) (29 points) & $-0.0451_{-0.0625}^{+0.0614}$ & $65.2299_{-2.4607}^{+2.4862}$ & - & \\
SN (Union2.1 ) & $-0.3077_{-0.1036}^{+0.1045}$ & $68.7702_{-1.3754}^{+1.4052}$ & - & This Letter \\
\hline \hline
\end{tabular}

Table 3. Numerical results summary of simulated data.

\begin{tabular}{lcccc}
\hline \hline Data & $\langle q\rangle$ & $\left\langle H_{0}\right\rangle(\mathrm{km} / \mathrm{s} / \mathrm{Mpc})$ & $\langle r\rangle$ & $\langle s\rangle$ \\
\hline \multirow{2}{*}{ JDEM } & $-0.0656_{+0.0003}^{-0.0003}$ & $25.9083_{+0.0030}^{-0.0030}$ & $-0.0570_{+0.0002}^{-0.0002}$ & $0.6229_{+0.0002}^{-0.0002}$ \\
& & & & \\
\hline \hline
\end{tabular}

well with $\mathrm{H}(\mathrm{z})$ data but in our case, by comparing the values of $\chi_{\delta}^{2}$ of power law and $\Lambda$ CDM models, we find that the power law cosmological model does not fit well with the latest $\mathrm{H}(\mathrm{z})$ data.

\section{- Union2.1 SN Data :}

We now put constraints on the above said parameters by using Type Ia supernova observation which is one of the direct probes for the cosmological expansion. SNe Ia are always used as standard candles for estimating the apparent magnitude $m(z)$ at peak brightness after accounting for various corrections, and are believed to provide strongest constraints on the cosmological parameters. In this investigation, we work with recently released Union2.1 compilation set of $580 \mathrm{SNe}$ Ia data points. For a standard candle of absolute magnitude $M$ and luminosity distance $d_{L}$, the apparent magnitude $m(z)$ is expressed as

$$
m=M+5 \log _{10}\left(\frac{d_{L}}{1 M p c}\right)+25
$$


where $M$ is constant for all SNe Ia. Equation (4.2) can be written as

$$
m=M+5 \log _{10} D_{L}(z)-5 \log _{10} H_{0}+52.38 \text {. }
$$

where

$$
D_{L}(z)=\frac{H_{0}}{c} d_{L}(z)
$$

The distance modulus $\mu(z)=m-M$ is given by

$$
\mu(z)=5 \log _{10} D_{L}(z)-5 \log _{10} H_{0}+52.38 .
$$

$D_{L}$, the Hubble free luminosity distance can now be expressed as

$$
D_{L}(z)=(1+z) \int_{0}^{z} \frac{H_{0}}{H\left(z^{*}\right)} d z^{*}=\frac{1}{q}\left[(1+z)-\frac{1}{(1+z)^{q-1}}\right] .
$$

$\chi^{2}$ can be defined as

$$
\chi_{S N}^{2}=\sum_{i j}\left(\mu(\exp )_{i}-\mu(o b s)_{i}\right) C_{i j}^{-1}\left(\mu(\exp )_{j}-\mu(o b s)_{j}\right)
$$

where $C_{i j}$ is the full covariance matrix [28]. Best fit values of the power law model parameters in the parametric space $\left(q>-1\right.$ and $\left.H_{0} \geq 0\right)$ with $1 \sigma$ error are obtained as $q=-0.3077_{-0.1036}^{+0.1045}$ and $H_{0}=68.7702_{-1.3754}^{+1.4052} \mathrm{~km} / \mathrm{s} / \mathrm{Mpc}$ together with $\chi_{\delta}^{2}=0.9464$. Similar results obtained for $\Lambda$ CDM model parameters are $\Omega_{0 m}=$ $0.2955_{-0.0587}^{+0.0655}$ and $H_{0}=69.5493_{-1.3885}^{+1.4039} \mathrm{~km} / \mathrm{s} / \mathrm{Mpc}$ together with $\chi_{\delta}^{2}=0.9431$. Here, we observe that values of $\chi_{\delta}^{2}$ of both the models are approximately equal. Therefore, we can say that power law cosmological model fits well with Union2.1 compilation data. The $1 \sigma$ (dark shaded) and $2 \sigma$ (light shaded) likelihood contours for both the models are shown in figure 2. Best fitted behaviour for power law cosmological model with SN data and error bars has been shown in panel (b) of figure 3. We also observe from Table 2 that the revised analysis of SN data gives much larger error bars on both $q$ and $H_{0}$ than that of reference [12]. This might have occurred due to taking into account the full covariance matrix of the latest SN data in the present analysis that was ignored in reference [12].

From the best fit values presented for power law model with $\mathrm{H}(\mathrm{z})$ data and SN data, we observe that there is a large discrepancy between the values of $q$, this might occur because in the present updated observational analysis we see that power law model fits well with $\mathrm{SN}$ data but not with $\mathrm{H}(\mathrm{z})$ data.

\section{- JDEM: Simulated SN data}

We use simulated dataset [36] for the future JDEM, supernova surveys having approximately 2300 supernovae in the redshift range $0-1.7$. The errors do not depend on redshift and equal for all SNe. We take $\sigma=0.13$ [37]. Using latest specifications, we generated 500 data sets as explained in [38], considering $\Lambda$ CDM model as our fiducial model with $\Omega_{0 m}=0.3$. For each of these experiments i.e. 


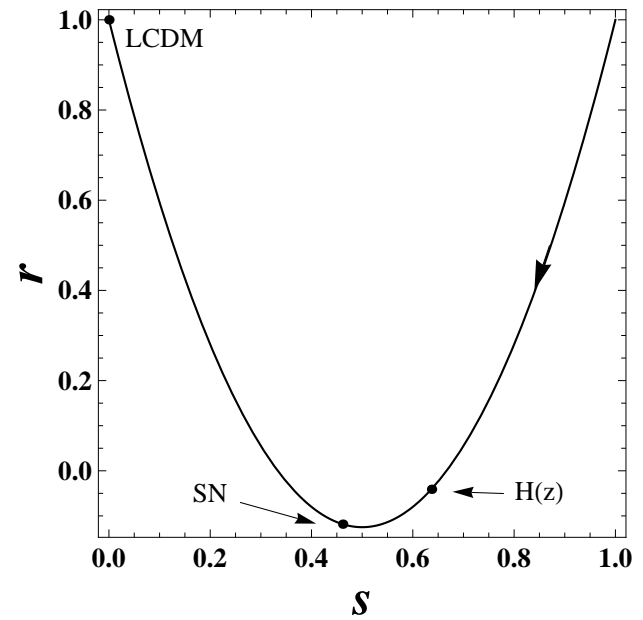

(a)

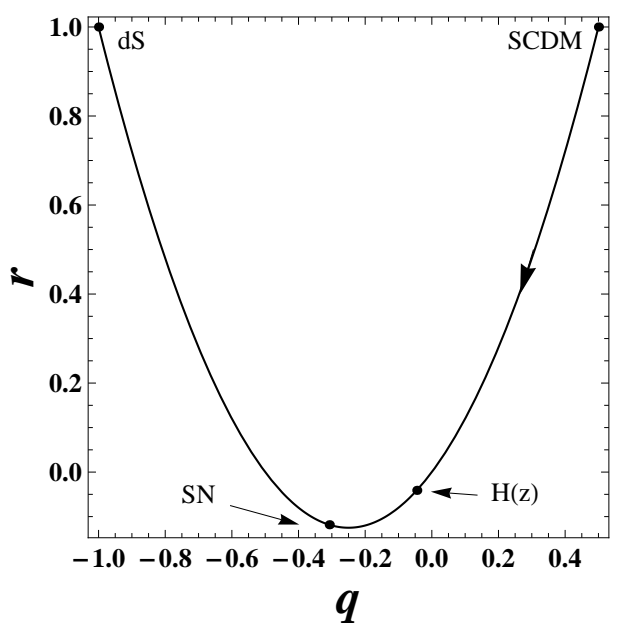

(b)

Figure 4. The panel (a) shows the time evolution of the statefinder pair $\{r, s\}$ for power- law cosmological model. The model converge to the fixed point $(r=1, s=0)$ which corresponds to LCDM. The panel (b) shows the time evolution of the statefinder pair $\{r, q\}$ for said model. The point $(r=1, q=0.5)$ corresponds to a matter dominated Universe (SCDM) and converges to the point $(r=1, q=-1)$ which corresponds to the de Sitter expansion $(\mathrm{dS})$. The black dots shown on the curves by arrows are the best fit values of $r, s$ and $q$ obtained by latest $\mathrm{H}(\mathrm{z})$ and $\mathrm{SN}$ data.

500 experiments, the best-fitting parameters $H_{0}$ and $q$ were calculated. We then calculated $r$ and $s$ for each experiment from the calculated values of the model parameters and finally we computed the mean values of $H_{0}, q, r$, and $s$ as $\left\langle H_{0}>\right.$, $\langle q\rangle,\langle r\rangle$, and $\langle s\rangle$. The numerical results obtained have been presented in Table 3.

\subsection{Constraints on Statefinders}

Following the analysis presented in reference [13], we obtain constraints on the statefinders with latest $\mathrm{H}(\mathrm{z})$ data as $r=-0.0410_{-0.0512}^{+0.0504}, s=0.6366_{-0.0417}^{+0.0409}$ and, with SN data as $r=-0.1183_{-0.0241}^{+0.0239}$ and $s=0.4615_{-0.0691}^{+0.0697}$. The above results have been summarized in Table 4.

We observe that power law model does not fit well with latest $\mathrm{H}(\mathrm{z})$ data and therefore significant changes in the best fit values are noticed from Table 2 and the corresponding variations in the constraints on statefinders can be observed from Table 4 but it fits well with updated SN data as well as previous data [12] therefore marginal differences are observed in the constraints from Table 2 and similar kind of variations can be noticed in statefinders from Table 4 . 
Table 4. Constraints on statefinders from latest $\mathrm{H}(\mathrm{z})$ and $\mathrm{SN}$ data.

\begin{tabular}{lccc}
\hline \hline Data & $r$ & $s$ & Refs. \\
\hline$H(z)$ (14 points) & $-0.09_{-0.03}^{+0.04}$ & $0.58_{-0.12}^{+0.04}$ & \\
SN (Union2) & $-0.09_{-0.02}^{+0.03}$ & $0.41_{-0.03}^{+0.03}$ & {$[12]$} \\
\hline$H(z)$ (29 points) & $-0.0410_{-0.0512}^{+0.0504}$ & $0.6366_{-0.0417}^{+0.0409}$ & \\
SN (Union2.1) & $-0.1183_{-0.0241}^{+0.0239}$ & $0.4615_{-0.0691}^{+0.0697}$ & This Letter \\
\hline \hline
\end{tabular}

\section{Conclusion}

Precision cosmological observations offer the possibility of uncovering essential properties of the Universe. Here, we have investigated power-law cosmology $a(t) \propto t^{\beta}$, which has some prominent features, making it unique when compared to other models of the Universe. For example, for $\beta \geq 1$, it addresses to the horizon, flatness and age problems $[17,18,20]$ and all these features provide viability to the power-law cosmology to dynamically solve the cosmological constant problem. In the work presented here, we used the most recent observational data sets from $\mathrm{H}(\mathrm{z})$ and SNe Ia observations and obtained the constraints on the two crucial cosmological parameters $H_{0}$ and $q$ and compared our results with reference [12]. We also have forecasted these constraints with simulated data for large future surveys like JDEM. Statistically, this model may be preferred over other models as we have to fit only two parameters. Numerical results obtained have been concluded in the Tables 2, 3 and 4 .

In this work, we observed that though $q$ is negative in the constraints from both $\mathrm{H}(\mathrm{z})$ and SNe Ia observations respectively but with bad $\chi_{\delta}^{2}$ in case of $\mathrm{H}(\mathrm{z})$ data, thus we can say that latter explains the present cosmic acceleration more efficiently than that of former in the context of power law cosmology. With the latest $\mathrm{H}(\mathrm{z})$ data, we found that obtained best fit value of $H_{0}$ for power law is outside more than $2 \sigma$ confidence level from the value of $\Lambda \mathrm{CDM}$ and also, we noticed the large discrepancy between the values of their $\chi_{\delta}^{2}$. In contrast, with $\mathrm{SN}$ data, we found that the value of $H_{0}$ for power law agrees with $\Lambda \mathrm{CDM}$ within $1 \sigma$ confidence level and also, its $\chi_{\delta}^{2}$ is approximately equal to the value of $\chi_{\delta}^{2}$ for $\Lambda \mathrm{CDM}$. Therefore, we conclude that power law model fits well with SN data but not with $\mathrm{H}(\mathrm{z})$ data. Contour plots for both the models with $\mathrm{H}(\mathrm{z})$ data and $\mathrm{SN}$ data have been shown in figures 1 and 2 respectively. Best fitted behaviour for power law model with data error bars have been shown in figure 3 . On comparing our results with reference [12], we observe that in the new analysis, best fit value of $q$ with $\mathrm{H}(\mathrm{z})$ data is drastically different from the constraint with $\mathrm{SN}$ data but it was not so significant in reference [12]. This discrepancy has been observed because 
in the current analysis, power law model does not fit well with latest $\mathrm{H}(\mathrm{z})$ data due to significant difference between the value of its $\chi_{\delta}^{2}$ and $\Lambda$ CDM. Also we observe that new SN analysis gives larger error bars on both $q$ and $H_{0}$ than that of reference [12] because of taking into account the full covariance matrix. Corresponding variations in the values of statefinders have also been observed which have been summarized in Table 4.

More explicitly, we see the differences in our study and of reference [12] as: in latter one it had been shown that the power law model fits well with both $\mathrm{H}(\mathrm{z})$ and SNe Ia observations but in our case we observed that it fits well only with SN data having larger error bars on both of the parameters and on the contrary it fails to fit with latest $\mathrm{H}(\mathrm{z})$ data shifting best fit value of $q$ significantly with bad $\chi_{\delta}^{2}$. Thus, we can say that our study explains merits and demerits of power law model in explaining the evolution of Universe in a more clear and sophisticated manner than that of reference [12]. The statefinder diagnostic carried out shows that power law cosmological model will finally approach the $\Lambda$ CDM model as shown in figure 4 . From the results mentioned in Table 3, one can also conclude that future surveys like JDEM demands an accelerated expansion of the Universe but with smaller values of Hubble constant within the framework of power law cosmology. From the above discussed results, it can be concluded that though power law cosmology has several prominent features but still it fails to explain redshift based transition of the Universe from deceleration to acceleration, because here we do not have redshift or time dependent deceleration parameter $q$. Thus, in nutshell it can clearly be said that despite having numerous remarkable features, the power law cosmology does not fit well in dealing with all cosmological challenges.

\section{Acknowledgment}

We are indebted to M. Sami for useful discussions and comments. Author SR thanks A. A. Sen, S. Jhingan and the whole CTP, JMI for providing the necessary facilities throughout this work. SR also acknowledges Gurmeet Singh and Vikas Kumar for their continuous support in improving this manuscript.

\section{References}

[1] V. Sahni and A. A. Starobinsky, Int. J. Mod. Phys. D 9, 373 (2000); E. J. Copeland, M. Sami, and S. Tsujikawa, (2006), Int. J. Mod. Phys., D15, 753; M. Sami, [arXiv:1401.7310]; M. Sami, R. Myrzakulov, [arXiv:1309.4188]; M. Sami, Curr. Sci. 97,887(2009) [arXiv:0904.3445]; M. Sami, [arXiv:0901.0756].

[2] D. Lohiya, M. Sethi , 1999, Class. Quantum Grav., 16, 1545

[3] A. Batra ,M. Sethi , D. Lohiya 1999, Phys. Rev. D, 60, 108301

[4] A. Batra, D. Lohiya, S. Mahajan, A. Mukherjee, 2000, Int. J. Mod. Phys. D, 9, 757

[5] S. Gehlaut, A. Mukherjee, S. Mahajan, D. Lohiya, 2002, Spacetime \& Substance, 4, 14

[6] S. Gehlaut, P. Kumar, G. Sethi, D. Lohiya, 2003, preprint (astro-ph/0306448) 
[7] A. Dev, D. Jain, D. Lohiya, 2008, preprint (astro-ph/0804.3491)

[8] A. Dev, M. Safanova, D. Jain, D. Lohiya, 2002, Phys. Lett. B, 548, 12

[9] G. Sethi, A. Dev, D. Jain, 2005, Phys. Lett. B, 624, 135

[10] Z.-H. Zhu, M. Hu, J.S. Alcaniz, Y.-X. Liu, 2008, A \& A, 483, 15

[11] B. Gumjudpai , K. Thepsuriya, Astrophys. Space Sci. 342, 537(2012)

[12] Suresh Kumar, MNRAS, 422, 2532-2538 (2012) [arXiv:1109.6924]

[13] B. Gumjudpai, [arXiv:1307.4552]

[14] C. Kaeonikhom , B. Gumjudpai, E.N. Saridakis, 2011, Phys. Lett. B, 695, 45

[15] A. D. Dolgov, (1982) in the The Very Early Universe, eds. G. Gibbons, S. Siklos, S. W. Hawking, (Eds.), Cambridge Univ. Press ; A. D. Dolgov, (1997), prd,55, 5881.

[16] L. H. Ford, (1987), prd, 35, 2339.

[17] P. Manheim, and D. Kazanas, (1990), grg, 22, 289.

[18] R. E. Allen, (1999), arXiv: astro-ph/9902042.

[19] S. Weinberg, ( 1989), Rev. Mod. Phys., 61,

[20] E. W. Kolb, (1989), apj, 344, 543.

[21] W.L. Freedman et al., 2001, ApJ, 553, 47

[22] S.H. Suyu et al., 2010, ApJ, 711, 201

[23] N. Jarosik et al., 2010, ApJS, 192, 14

[24] A.G. Riess et al., 2011, ApJ, 730, 119

[25] F. Beutler et al., 2011, MNRAS, 416, 3017

[26] P. A. R. Ade et al. [Planck Collaboration], arXiv:1303.5076 [astro-ph.CO].

[27] O. Farooq and B. Ratra, Astrophys. J. 766, L7 (2013) [arXiv:1301.5243 [astro-ph.CO]].

[28] N. Suzuki, D. Rubin, C. Lidman, G. Aldering, R. Amanullah, K. Barbary, L. F. Barrientos and J. Botyanszki et al., Astrophys. J. 746, 85 (2012) [arXiv:1105.3470 [astro-ph.CO]].

[29] C. Zhang, H. Zhang, S. Yuan, T. -J. Zhang and Y. -C. Sun, arXiv:1207.4541 [astro-ph.CO].

[30] J. Simon, L. Verde and R. Jimenez, Phys. Rev. D 71, 123001 (2005) [astro-ph/0412269].

[31] M. Moresco, L. Verde, L. Pozzetti, R. Jimenez and A. Cimatti, JCAP 1207, 053 (2012) [arXiv:1201.6658 [astro-ph.CO]].

[32] C. -H. Chuang and Y. Wang, arXiv:1209.0210 [astro-ph.CO].

[33] C. Blake, S. Brough, M. Colless, C. Contreras, W. Couch, S. Croom, D. Croton and T. Davis et al., Mon. Not. Roy. Astron. Soc. 425, 405 (2012) [arXiv:1204.3674 [astro-ph.CO]].

[34] D. Stern, R. Jimenez, L. Verde, M. Kamionkowski and S. A. Stanford, JCAP 1002, 008 (2010) [arXiv:0907.3149 [astro-ph.CO]]. 
[35] N. G. Busca, T. Delubac, J. Rich, S. Bailey, A. Font-Ribera, D. Kirkby, J. M. Le Goff and M. M. Pieri et al., Astron. Astrophys. 552, A96 (2013) [arXiv:1211.2616 [astro-ph.CO]].

[36] Holsclaw T. et al., 2010, Phys.Rev.Lett 105, 241302.

[37] Aldering G., et al. [SNAP Collaboration], 2004, arXiv:astro-ph/0405232

[38] Alam U., Sahni V., Saini T.D., Starobinsky A.A., 2003, MNRAS, 344, 1057

[39] Malekjani M., Khodam-Mohammadi A., 2012, preprint arxiv:1202.4154v1

[40] Sahni V., Saini T.D., Starobinsky A.A., Alam U., 2003, Pisma Zh. Eksp. Teor. Fiz., 77,249

[41] Sahni V., 2002, preprint (astro-ph/0211084)

[42] M. Sami, M. Shahalam, M. Skugoreva, A. Toporensky, Phys. Rev. D 86, 103532 (2012) [arXiv:1207.6691].

[43] Ozgur Akarsu, Suresh Kumar, R. Myrzakulov, M. Sami, Lixin Xu, JCAP 01 (2014) 022 [arXiv:1307.4911]; Ozgur Akarsu, Tekin Dereli, Suresh Kumar, Lixin Xu, EPJP 129 (2014) 22 [arXiv:1305.5190].

[44] R. Myrzakulov and M. Shahalam, JCAP 10 (2013) 047 [arXiv:1303.0194]; L.P. Chimento, A. S. Jakubi, D. Pavon and W. Zimdahl, Phys.Rev. D 67, 083513 (2003) [astro-ph/0303145]; L. Zhang, J. Cui, J. Zhang, and X. Zhang, Int. J. Mod. Phys. D 19, 21 (2010) [arXiv:0911.2838]; A. Khodam-Mohammadi and M. Malekjani, Astrophys. Space Sci.331:265-273 (2011) [arXiv:1003.0543]; Z. L. Yi and T. J. Zhang, Phys.Rev.D75, 083515 (2007) [astro-ph/0703630]; M. Tong, Y. Zhang and T. Xia, Int. J.Mod. Phys.D18, 797 (2009) [arXiv:0809.2123]; H. Farajollahi and A. Salehi, JCAP 1011:006 (2010) [arXiv:1010.3589]; 